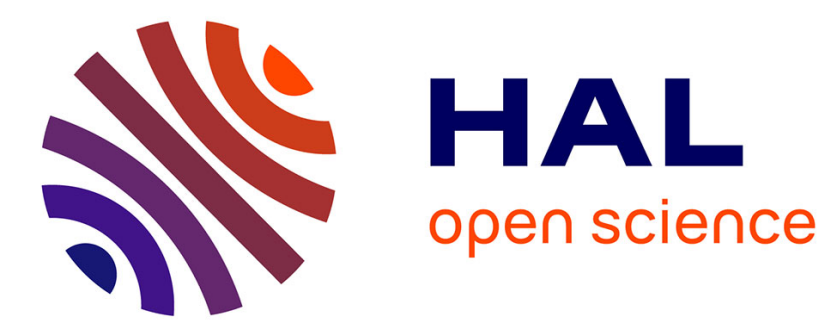

\title{
HOT ELECTRON NOISE PROPERTIES OF SEMICONDUCTORS IN THE NON-ZERO COLLISION DURATION REGIME
}

\author{
P. Das, D. Ferry, H. Grubin
}

\section{> To cite this version:}

P. Das, D. Ferry, H. Grubin. HOT ELECTRON NOISE PROPERTIES OF SEMICONDUCTORS IN THE NON-ZERO COLLISION DURATION REGIME. Journal de Physique Colloques, 1981, 42 (C7), pp.C7-227-C7-234. 10.1051/jphyscol:1981727 . jpa-00221664

\section{HAL Id: jpa-00221664 https://hal.science/jpa-00221664}

Submitted on 1 Jan 1981

HAL is a multi-disciplinary open access archive for the deposit and dissemination of scientific research documents, whether they are published or not. The documents may come from teaching and research institutions in France or abroad, or from public or private research centers.
L'archive ouverte pluridisciplinaire HAL, est destinée au dépôt et à la diffusion de documents scientifiques de niveau recherche, publiés ou non, émanant des établissements d'enseignement et de recherche français ou étrangers, des laboratoires publics ou privés. 


\title{
HOT ELECTRON NOISE PROPERTIES OF SEMICONDUCTORS IN THE NON-ZERO COLLISION DURATION REGIME ${ }^{¥}$
}

\author{
P. Das ${ }^{\text {XII }}$, D.K. Ferry* and H. Grubin ${ }^{*}$ \\ Rensselaew Polytechnic Institute, Troy, NY 12181, U.S.A. \\ *Colorado State University, Fort Colzins, CO 80523, U.S.A. \\ * Scientific Research Associates, Glastonbury, CT 06033, U.S.A.
}

\begin{abstract}
Résumé.- Pour caractériser le bruit de composants en régime d'électrons chauds, on résoud l'équation de Langevin en incluant les effets du temps de relaxation de l'énergie et du moment dans l'approximation d'une distribution maxwellienne déplacée. On tient aussi compte de la durée de collision qui peut être une fraction non négligeable du temps de relaxation de l'énergie et du moment. On sait que la densité spectrale de puissance de bruit et par suite le coefficient de diffusion (en petits signaux) et la fonction d'autocorrélation de la vitesse sont reliées à la conductivité microondes des semiconducteurs (pour les petits signaux). Les composantes transversales et longitudinales de ces grandeurs sont calculées en incluant leur dépendance de la durée de la collision. Un calcul numérique utilisant les constantes du silicium a èté développé et montre la. contribution importante des durées de collision non nulles.
\end{abstract}

Abstract.- To obtain the noise properties of devices under hot electron condition, Langevin's equation is solved including the effects of energy and momentum relaxation time in the displaced maxwellian distribution approximation. The collision duration, which can be a significant fraction of energy or momentum relaxation time for very small structured devices, is also included. It is known that the noise power spectral density and thus the small signal diffusion coefficient and velocity auto-correlation are related to the small signal microwave conductivity of semiconductors. Both the transverse and longitudinal components of these quantities are calculated including their dependence on the magnitude of collision duration. Numerical calculation using the constants of silicon has been performed and it shows the significant contribution of the nonzero collision duration.

\section{INTRODUCTION}

It is we11-known that the microwave conductivity of semiconductors varies as a function of frequency and that this functional dependence becomes quite complicated when hot electron transport is included [1]. This occurs because one must consider not only the momentum relaxation time of the carriers but also the energy relaxation time and any consequent differential repopu-

\footnotetext{
\# Partially supported by ONR, NSF and AFOSR

** On leave at the Electrical Engineering and Computer Science Department, University of California at San Diego, U.S.A.
} 
lation in many valley systems [2-4]. In particular, enhanced conductivity arises at high frequencies due to the process of velocity overshoot. In small semiconductor devices, the time scale of carrier transport through the device, with the expected high fields present, is such that the device dynamics may well be dominated by the transient response characteristics of the carrier velocity and distribution function [5]. However, if this becomes the case, major modifications are required to the Boltzmann transport equation [6] and to the current response equations within the devices [7,8]. The fact that the relaxation times are varying, due to the evolving of the average energy, on the same time scale appropriate to the velocity response and that a finite, non-zero collision duration exists both lead to a complicated, multiply-convolved form for the transport balance equations [8].

It is also known that the noise properties of semiconductors are related to the hot electron microwave conductivity $[9,10,11,12]$. For an example, the noise power spectrum is proportional to the real part of the microwave conductivity. As the noise power spectrum is related to the velocity auto-correlation through Fourier transform and also to diffusion co-efficient, a11 these qantities can be obtained from the knowledge of microwave conductivity as a function of frequency. Recently this microwave conductivity has been calculated in the non-zero collision region [13], applicable for a single valley semiconductor. The purpose of the present paper is to extend the calculations to multivalley case and relate them to the noise power spectrum, velocity auto-correlation and diffusion co-efficient through the solution of Langevin's equation.

\section{MICROWAVE CONDUCTIVITY}

\section{i) Parallel polarization case}

Let us consider that the semiconductor is under the influence of a d.c. bias electric field, $F_{0}$, in addition to the microwave field given by $F_{7} e^{j \omega t}$ where $F_{1} / F_{0}<1$ and $\omega$ is the angular frequency of the microwave field. For the parallel polarization case we assume that the direction of $F_{1}$ and $F_{0}$ is collinear. Using the formulation outlined in ref. 13 it is easy to show the following equations for the perturbed drift velocity $v_{1} e^{j \omega t}$ in the presence of a d,c. value $v_{0}$. Before we write down the equations we note that the inclusion of a non-zero collision time, $\tau_{c}$, does not change the final equilbrium value, $v_{0}$. Also the only change in the momentum and energy balance equations imposed by $\tau_{c}$ is to change the momentum relaxation time $\tau_{m}$ by $\tau_{m}\left(1+j \omega \tau_{c}\right)$ and the energy relaxation time, $\tau_{e}$ by $\tau_{e}\left(1+j \omega \tau_{c}\right)$. Thus for a single valley case, one obtains

$$
j \omega v_{1}=-\frac{m v_{1} \Gamma_{m o}}{i+j \omega \tau_{c}}-\frac{m v_{0} \Gamma_{m}^{\prime} T_{1}}{i+j \omega \tau}+\frac{e F_{1}}{m} \quad \begin{aligned}
& \text { (momentum } \\
& \text { balance), }
\end{aligned}
$$




$$
j \omega k T_{1}=-\frac{k T_{1} \Gamma_{e 0}}{1+j \omega \tau_{c}}-\frac{k T_{0} \Gamma^{\prime}}{1+j \omega \tau_{c}}+\frac{2 e}{3}\left(v_{0} F_{1}+v_{1} F_{0}\right) \text { (energy balance) }
$$

$m$ is the effective mass of the carriers, $k$ is the Boltzmann's constant, $T$ is the effective temperature in the displaced Maxwellian distribution approximation and

$$
\begin{aligned}
& \frac{1}{\tau_{m}}=\Gamma_{m}=\Gamma_{m o}+\Gamma_{m}^{\prime} T_{1} e^{j \omega t} \\
& \frac{1}{\tau_{e}}=\Gamma_{e}=\Gamma_{e o}+\Gamma_{e}{ }^{\prime} T_{1} e^{j \omega t}
\end{aligned}
$$

The subscript "o" denotes the equilibrium value and the prime denotes the differentiation with respect $T$ taken at $T=T_{0}$. Both $\tau_{m}$ and $\tau_{e}$ are effective relaxation times which include the effects of all possible forms of scattering mechanisms relevant for the case (e.g. acoustic, optical, intervalley phonons, impurity scattering etc.).

For the two-valley case, following ref. 14, one obtains the following modified equations,

$$
\begin{aligned}
& j \omega v_{1 i}=-\frac{m v_{1 i} \Gamma_{m o i}}{1+j \omega T_{c i}}-\frac{m v_{0}{ }_{m i}^{1} T_{1 i}}{1+j \omega \tau_{c i}}+\frac{e F_{1}}{m_{i}} \quad \text { (momentum ba } 1 \text { ance) } \\
& j \omega k T_{7 i}=-\frac{k T_{1 i} \Gamma_{e o i}}{T+j \omega \tau_{c i}}-\frac{k T_{0 i}{ }^{\prime}{ }^{\prime} T_{l i}}{1+j \omega \tau_{c i}}+\frac{2 e}{3}\left(v_{0} F_{1}+v_{1} F_{0}\right) \\
& +\frac{n_{j 0} k \theta \Gamma_{i j o}}{n_{i 0}\left(1+\omega \tau_{c j}\right)}\left[\frac{n_{j l}}{n_{j 0}}-\frac{n_{i 1}}{n_{i 0}}+\frac{\Gamma_{i j}^{\prime} T_{1 j}}{\Gamma_{i j o}}\right] \\
& \text { (energy balance) } \\
& j \omega n_{i 1}=-n_{i 1} \Gamma_{n i}-n_{i 0} \Gamma_{n i}^{\prime}+n_{j i} \Gamma_{n j}+n_{j 0} \Gamma_{n j}^{\prime} \\
& n_{11}+n_{21}=0 \text {. }
\end{aligned}
$$

The subscripts $i, j=1,2$ and denotes the various parameters for the two valleys, $\tau_{n i}$ and $\tau_{i j}$ denotes the relaxationtimes associated with the number balance and intervalley phonon respectively,

$$
\begin{aligned}
& \Gamma_{n i}=\frac{1}{\tau_{n i}} \\
& \Gamma_{i j}=\frac{1}{\tau_{i j}} .
\end{aligned}
$$

The quantities $\Gamma_{i j}, \Gamma_{n j}, \Gamma_{i j}^{\prime}$ and $\Gamma_{n j}^{\prime}$ are functions of $T_{j}$ only. $k \theta$ is the characteristic phonon temperature used in equ. (4) only for convenience.

Equations (3-5) can be solved to obtain an analytical expression for the effective microwave conductivity, $\mu_{11}$. However, it is rather cumbersome although straightforward and will not be given here. For numerical calculations it is better to tackle the equations directly. The above equations 
can also be extended in a rather straightforward manner for the multivalley case having more than two valleys.

For the single valley case, however, one obtains

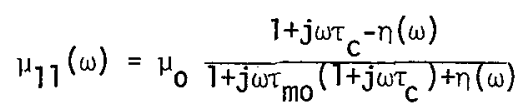

where $\mu_{0}=\frac{\mathrm{e}}{\mathrm{m} \Gamma_{\mathrm{mo}}}$

and

$$
n(\omega)=\left(1+j \omega \tau_{c}\right) \frac{T_{e o}}{\gamma(\omega) \Gamma_{m o}} \Gamma_{m}^{\prime}
$$

$$
\gamma(\omega)=1+\frac{T_{e o}}{\Gamma_{e o}} \quad \Gamma_{e}^{\prime}+\frac{3}{2}{ }^{j \omega \tau_{e o}}\left(1+j \omega \tau_{c}\right)
$$

\section{ii) Perpendicular polarization case.}

If $F_{1}$ is normal to $F_{0}$ then the perturbation in effective temperature is zero for first order calculations. Thus for this case equ. (1) simplies to

$$
j \omega v_{1}=-\frac{v_{1} \Gamma_{m o}}{i+j \omega \tau_{c}}+\frac{e F_{1}}{m}
$$

From this we immediately obtain

$$
\mu_{\perp}(\omega)=\mu_{0} \frac{1+j \omega \tau c}{1-\omega^{2} \tau_{m o} \tau_{c}+j \omega \tau \text { mo }}
$$

For the two valley case one obtains for each individual valley, an expression for mobility given by equ. (8). The microwave conductivity for the two valley case is given by

$$
\mu_{\perp}(\omega)=\frac{n_{10} \mu_{\perp 1}(\omega)+n_{20} \mu_{\perp 2}(\omega)}{n_{10}+n_{20}}
$$

\section{HOT ELECTRON NOISE PROPERTIES.}

To obtain hot electron noise properties one need to solve the Langevin's equation. For this purpose one assumes that a bias electric field is applied across the semiconductor in addition to the small fluctuating random electric field. Thus the difference between the problem solved in the last section and that of Langevin's equation is that one should consider $F_{1}$ as the spectral component of the fluctuating electric field. As the equations (1)-(6) are linear equations around a bias electric field, the solution of the Langevin's equation is equivalent to that discussed in references [ 9 and 12]. The noise spectral density for the equivalent noise voltage $\Phi(\omega)$

for the single valley case is given by for the parallel polarization case

$$
\phi(\omega)=\operatorname{A} 2 k T\left(n_{0} e\right) \operatorname{Re} \mu_{11}(\omega)
$$


where $A$ includes the dimensional factors. For the perpendicular polarization case $\mu_{11}$ is replaced by the $\mu_{\perp}$. Applying Weiner-Khintchin theorem one obtains the velocity auto-correlation, $\langle v(t) v(t+\tau)\rangle$, to be given by

$$
<v(t) v(t+\tau)>\propto F^{-1}\left[\mu_{r}(\omega)\right]
$$

where $F^{-1}$ denotes inverse fourier transform and $\mu_{r}(\omega)$ is the real part of the microwave mobility. As the diffusion co-efficient $D(\omega)$ is related to the Fourier transform of velocity auto-correlation, one obtains its value from the extended Einstein relationship

$$
R_{e} D(\omega)=\frac{k T}{q} \mu_{r}
$$

where $\mathrm{R}_{\mathrm{e}}$ denotes the real part.

Numerical results and discussion

The small signal microwave conductivity was calculated for Si for an applied d.c. field at $30 \mathrm{KvCm}^{-1}$. The scattering mechanisms and coupling constants are those used previously for Si [15-17]. The real and imaginary part of $\mu$ are defined as

$$
\mu=\mu_{r}+j \omega \mu_{I}
$$

and are plotted in fig. 1 and 2 for the parallel and perpendicular polarization cases respectively. The peaking at high frequencies is more pronounced in the presence of the non-zero collision duration, a result expected from calculations of overshoot velocity-itself.

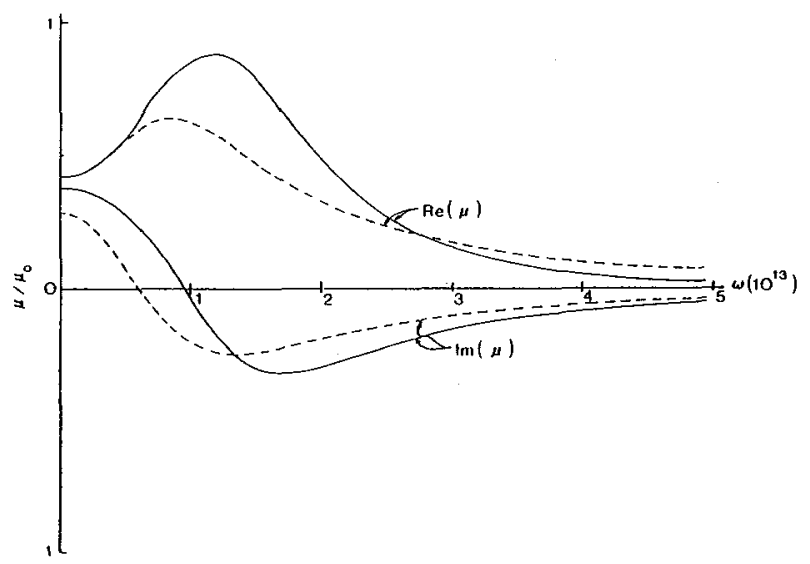

Fig. 1. Parallel Polarization: The real (a) and imaginary (b) parts of the a.c. small signal mobility for an applied d.c. field of $30 \mathrm{kV} \mathrm{cm}^{-1}$ in Si. The solid curve includes the effect of a finite, non-zero collision duration, while the dashed curve ignores this effect. 
The peaking observed leads to an interesting shift of the apparent plasma edge in the semiconductor. In fig. 3 we plot the reflectivity $|R|$ defined below for the parallel polarization case.

$$
|R=| \frac{\epsilon^{\frac{1}{2}}-1}{\epsilon^{\frac{1}{2}}+1} \mid
$$

where

$$
\epsilon=\epsilon_{s}\left[1-j \frac{n e^{2} \mu(\omega)}{\omega \varepsilon_{s} \varepsilon_{0}}\right]
$$

and $\varepsilon_{\mathrm{S}}$ is the static dielectric constant of the semiconductor and $\varepsilon_{0}$ is vacuum permittivity. Three curves are shown in fig. 3 . The

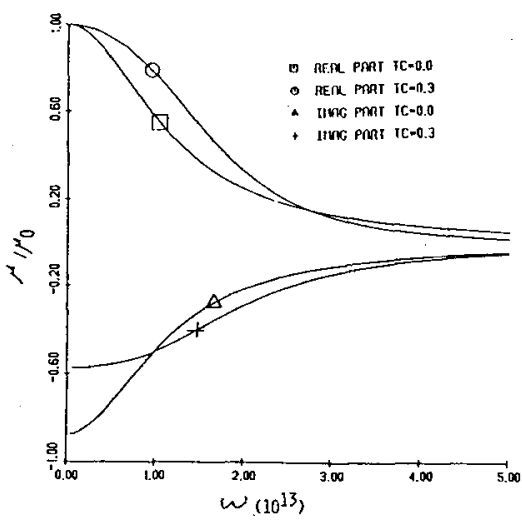

Fig. 2 Perpendicular Polarization: The real (a) and imaginary (b) parts of the a.c. small signal mobility for an applied d.c. field of $30 \mathrm{kV} \mathrm{cm}^{-1}$ in Si.

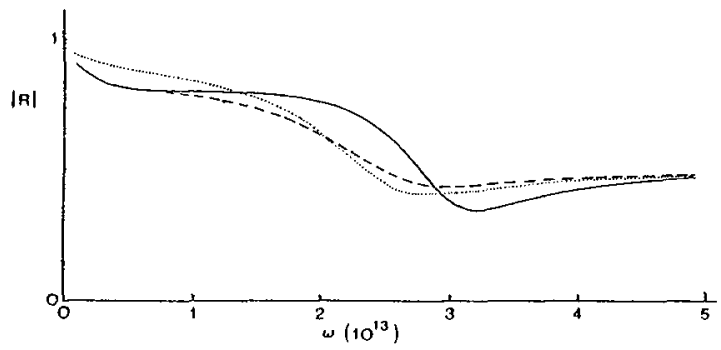

Fig. 3. The reflectivity of $\mathrm{Si}$ in a high electric field. The dotted curve assumes cold carriers with mobility $\mu_{0}$, but a different chordal mobility. The solid curve includes the effect of the finite collision duration. A doping of $1018 \mathrm{~cm}^{-3}$ is assumed. 
dotted curve assumes cold carriers with mobility $\mu_{0}$ while the dashed curve assumes hot carriers with the same $\mu_{0}$ but a different chordal mobility. The solid curve includes the effect of the finite collision duration. A doping of $10^{18} \mathrm{~cm}^{-3}$ is assumed. It is observed that the presence of hot carriers merely serves to smooth the apparent plasma edge. However, for $\tau_{c} \neq 0$, the minimum shifts significantly to higher frequencies.

The velocity auto-correlations obtained by directly Fourier transforming $\mu_{r}(\omega)$ is shown in figs. 4 and 5 for the two polarization cases. As expected, it is observed that the inciusion of non zero collision duration contributes significantly to the negative swing of the velocity auto-correlation.

In conclusion, the small signal microwave conductivity of semiconductors in the hot electron condition has been obtained in the nonzero collision regime which in turn has been used to obtain noise properties.

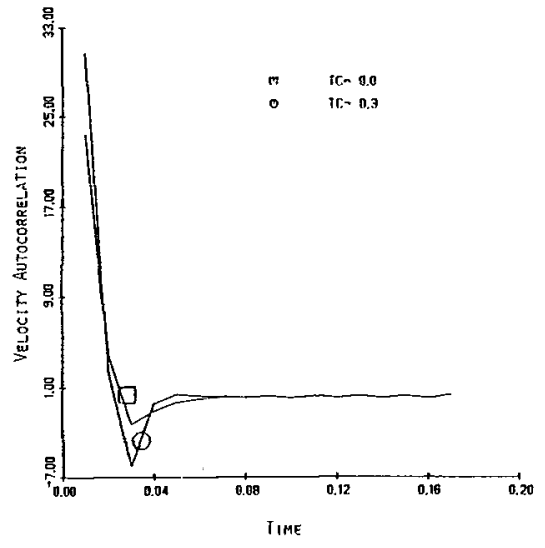

Fig. 4. Velocity auto-correlation for the parallel case. Units are arbitrary.

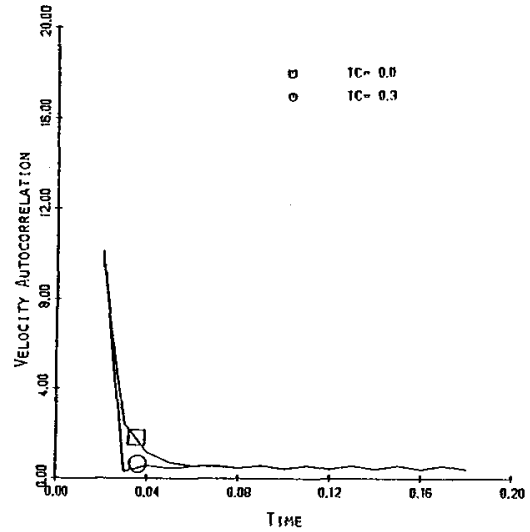

Fig. 5. Velocity auto-correlation for the perpendicular case. Units are arbitrary.

\section{REFERENCES}

[1] P. Das \& D. K. Ferry, Solid State Electron, 19, 851 (1976).

[2]- D. K. Ferry \& P. Das, Solid State Electron, 20, 355 (1977).

[3] H. D. Rees, IBM J. Res. Develop, 13, 537 (1969).

[4] H. L. Grubin, D.K. Ferry \& J. R. Barker, Proc. IEDM, P. 394 (1979).

[5] D. K. Ferry \& J. R. Barker, Solid State Electron, 23, 545 (1980). 
[6] J. R. Barker \& D. K. Ferry, Solid State Electron, 23, 519 (1980).

[7] D. K. Ferry \& J. R. Barker, Solid State Commun. 30, 361 (1979).

[8] D. K. Ferry \& J. R. Barker,J. Phys. Chem. Solids (in press).

[9] A. Van Der Ziel, "noise in Solid State Devices", in Advances in Electronics and Electron Physics, Ed. L. Harton, Vol. 46, Academic Press, (1978).

[10] J. P. Nougier, D. Sodini, M. Rolland, D. Gasquet and Gasquet and G. Lecoy, Solid State Electron, 21, 133 (1978).

[11] A. Van Der Ziel, Solid State Electronics, Vol. 23, pp. 1035-1036 (1980).

[12] C. W. Helstrom, "Markov Processes and applications", in Communcation Theory", Ed. A. V. Balakrishnar, McGraw Hil1, (1968).

[13] P. Das, D. K. Ferry and H. Grubin, Solid State Commun., to be published.

[14] P. Das, App1. Phys. Letters, Vo1. 11, pp-386-388 (1967).

[15] D. K. Ferry, Phys. Rev. B14, 1605, (1976).

[16] D. K. Ferry, Surf. Sci., 57, 218 (1976).

[17] D. K. Ferry, Phys. Rev. B.14, 5364 (1976). 